\title{
MET Positive
}

National Cancer Institute

\section{Source}

National Cancer Institute. MET Positive. NCI Thesaurus. Code C131068.

Indicates that MET expression has been detected in a sample. 